AperTO - Archivio Istituzionale Open Access dell'Università di Torino

\title{
A growth model for multicellular Tumor Spheroids
}

\section{This is a pre print version of the following article:}

Original Citation:

\section{Availability:}

This version is available http://hdl.handle.net/2318/36884

since

Terms of use:

Open Access

Anyone can freely access the full text of works made available as "Open Access". Works made available under a Creative Commons license can be used according to the terms and conditions of said license. Use of all other works requires consent of the right holder (author or publisher) if not exempted from copyright protection by the applicable law. 


\title{
Growth model for multicellular tumor spheroids
}

\author{
Pier Paolo Delsanto a) \\ Dip Fisica, Politecnico di Torino and Bioindustry Park Canavese, Colleretto Giacosa, Torino, Italy
}

Caterina Guiot and Piero Giorgio Degiorgis

Dip. Neuroscience, Università di Torino, 10125 Torino, Italy, and INFM, sezioni di Torino Università e Politecnico, Italy

Carlos A. Condat

Department of Physics, University of Puerto Rico, Mayagüez, PR 00681, and CONICET and FaMAF, Universidad Nacional de Córdoba, 5000-Córdoba, Argentina

Yuri Mansury

Complex Biosystems Modeling Laboratory, Harvard-MIT (HST) Athinoula A. Martinos Center for Biomedical Imaging, HST-Biomedical Engineering Center, Massachusetts Institute of Technology, Cambridge, Massachusetts 02139

Thomas S. Deisboeck

Complex Biosystems Modeling Laboratory, Harvard-MIT (HST) Athinoula A. Martinos Center for Biomedical Imaging, HST-Biomedical Engineering Center, Massachusetts Institute of Technology, Cambridge, Massachusetts 02139 and Molecular Neuro-Oncology Laboratory, Harvard Medical School, Massachusetts General Hospital, Charlestown, Massachusetts 02129

(Received 5 April 2004; accepted 2 September 2004)

\begin{abstract}
Most organisms grow according to simple laws, which in principle can be derived from energy conservation and scaling arguments, critically dependent on the relation between the metabolic rate $B$ of energy flow and the organism mass $m$. Although this relation is generally recognized to be of the form $B(m)=m^{p}$, the specific value of the exponent $p$ is the object of an ongoing debate, with many mechanisms being postulated to support different predictions. We propose that multicellular tumor spheroids provide an ideal experimental model system for testing these allometric growth theories, especially under controlled conditions of malnourishment and applied mechanical stress. (C) 2004 American Institute of Physics. [DOI: 10.1063/1.1812842]
\end{abstract}

It was recently proposed ${ }^{1}$ that tumors may follow the general model of ontogenetic growth for all living organisms (from mammals to mollusks to plants) developed by West, Brown and Enquist (WBE). ${ }^{2}$ The beauty of the WBE model is that it is entirely based on energy conservation and other general physical arguments, i.e., scaling. The authors start with the assumption that the energy intake, supplied by ingested nutrients, is spent partly to support the metabolic functions of the organism's existing cells and partly for cell replication, i.e., to reproduce new cells. Based on this key assumption, a universal growth curve is derived, which the authors conjectured to be applicable to all living organisms. Their conjecture, based on a fractal-like distribution model, is supported by data encompassing many different species. ${ }^{2}$ Although they assumed $B \propto m^{p}$ with $p=3 / 4$, their value of $p$ is not universally accepted. Indeed there is strong evidence that growth may be consistent with a $2 / 3$ law in the case of birds and small mammals. ${ }^{3}$ Due to its implications for tumor metastasis, cell turnover rates, angiogenesis, and invasion, the proposal of Ref. 1 has immediately contributed to an ongoing debate. ${ }^{4,5}$

Many different explanations have been put forward for the scaling laws, ranging from four-dimensional biology. ${ }^{6}$ and quantum statistics ${ }^{7}$ to long-bone allometry combined with muscular development, ${ }^{8}$ but, as yet, the debate is far from being settled. In this letter we suggest that multicellular

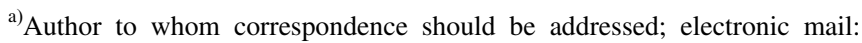
caterina.guiot@unito.it
}

tumor spheroids (MTS) are excellent experimental model systems to test the validity of the proposed mechanisms. MTS are spherical aggregations of malignant cells, ${ }^{9,10}$ which can be grown in vitro under strictly controlled nutritional and mechanical conditions. Although cells appear to preserve the evolutionary self-organization rules governing the growth of the original tumor line, they also evolve according to the environmental conditions of the particular experimental setting. Here we show that MTS indeed grow following a scaling law and obtain their expected properties under conditions of malnourishment and rising mechanical stress-conditions that often apply to tumors in vivo.

Following WBE, we assume, for simplicity, that metabolism and growth are the same for all MTS cells. Let $B$ be the resting metabolic energy expenditure. Then, at any time $t$ and for any discrete short time interval $\Delta t$, the law of energy conservation yields

$$
B \Delta t=N \beta \Delta t+\varepsilon \Delta N
$$

where $N$ is the total number of cells, $\beta$ is the metabolic rate for a single cell, and $\varepsilon$ is the energy required to create a new cell. Since in general volumetric, rather than mass, measurements are performed on MTS, we rewrite Eq. (1) in terms of volumes (assuming constant cell density). Let $\gamma$ be the volume of a single cell and $v=N \gamma$ the volume of the MTS. Writing $B=B_{0} v^{p}$, for a general choice of the exponent $p$, Eq. (1) becomes 


$$
\frac{d v}{d t}=a v^{p}-b v
$$

where $a=\gamma B_{0} / \varepsilon$ and $b=\beta / \varepsilon$. It follows that, starting from a volume $v_{0}$ at birth, $v$ increases up to a maximum value $V$ $=(a / b)^{1 /(1-p)}$, corresponding to the zero growth point $d v / d t$ $=0$.

Note that Eq. (2) follows von Bertalanffy's allometric law, which has been used to describe growth processes. ${ }^{11} \mathrm{~A}$ number of alternative specifications, such as the logistic and Gompertzian laws, have been previously used to fit spheroid growth data. ${ }^{11}$.

Introducing the function

$$
y(t)=1-\left(\frac{v(t)}{V}\right)^{1-p},
$$

Eq. (2) becomes

$$
\frac{d y}{d t}=-\alpha y \text {, }
$$

with $\alpha=b(1-p)$. If we now define $\mathrm{e}^{2}$ the dimensionless variable $\tau=(1-p) b t-\ln \left[1-\left(v_{0} / V\right)^{1-p}\right]$, Eq. (4) has the solution $y(\tau)=e^{-\tau}$, which does not depend on the choice of $p$ and, therefore, is universally valid.

Since effective nutrient absorption cannot start immediately after spheroid implantation into the experimental surrounding; we model this start-up phase or "accommodation time" by replacing the parameter $a$ in Eq. (2) with the timedependent function $a_{1}(t)=a\left(1-e^{-t / T}\right)$, where $T$ is the effective accommodation time.

Let us now turn our attention to the impact of nutrients replenishment on MTS growth. Data from Freyer and Sutherland. ${ }^{12}$ show that when the nutrient content of the tissue culture medium is depleted (in particular of glucose and oxygen), the growth curve of the spheroids is modified, i.e., it reaches a volume lower than the asymptotic value $V$. The condition of insufficient nutrient availability can be reproduced in our model by the subtraction of a term in Eq. (2) representing the missing power. It is plausible to write such a term as $f \alpha v^{p}$, with the fraction $f \in[0,1]$ and $f=0$ in conditions of full nutrient availability. Then Eq. (2) becomes

$$
\frac{d y}{d t}+\alpha y=\alpha f+\alpha(1-f) e^{-t / T} .
$$

Equation (5) has a simple exact analytical solution whose asymptotic value $y(\infty)=f$ corresponds to the asymptotic volume

$$
V_{\infty}=V(1-f)^{1 /(1-p)}(0 \leqslant f<1) .
$$

Figure 1 shows experimental data from Ref. 12 for different degrees of undernourishment. Specifically, these authors cultured EMT6/Ro mouse mammary carcinoma cell spheroids in suspension with various oxygen and glucose concentrations and measured spheroid growth saturation sizes as well as viable rim thickness. Both correlated positively with the oxygen and glucose concentrations in the medium. Since the available data do not allow us to select an optimal value for $p$, we choose $p=2 / 3$ (which would correspond to diffusioncontrolled nutrient inflow) as our working assumption for the fits (solid lines). The agreement is excellent, except for the case of most severe undernourishment $(f=0.8)$. This is not surprising, because a strong nutrient deficiency should also

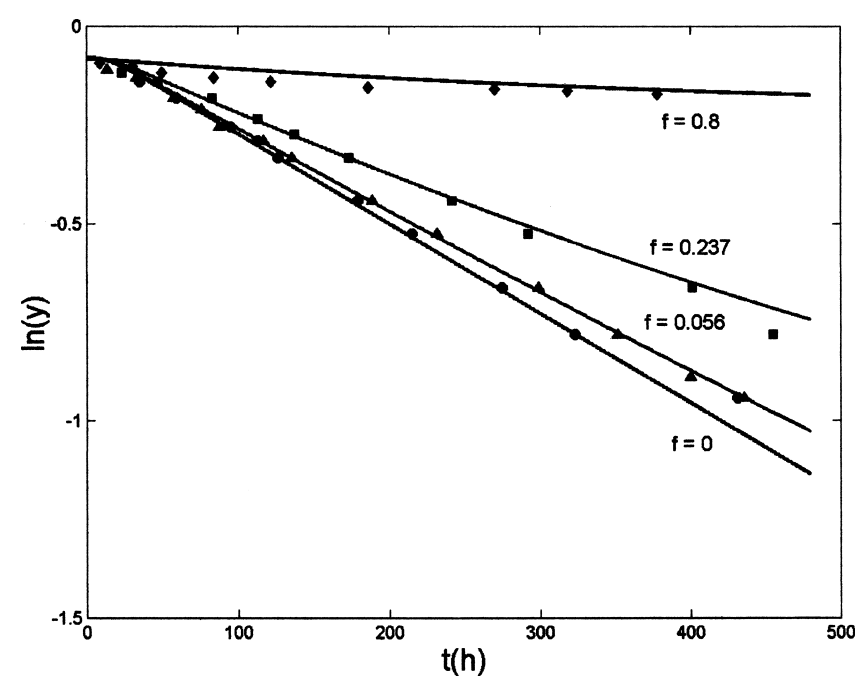

FIG. 1. Time variation of the undernourished spheroid mass [see Eq. (3), data from Ref. 12]. Solid curves are model fits for a choice of $p=2 / 3$. The $y$ intercept corresponds to $v_{0}=2 \times 10^{-6} \mathrm{~cm}^{3}$. Final volumes $V_{\infty}$ are, starting from the lowest curve: $4.4 \times 10^{-3}, 3.7 \times 10^{-3}, 1.95 \times 10^{-3}$, and 3.56 $\times 10^{-5} \mathrm{~cm}^{3}$. The accommodation time is, in all cases, $T=10 \mathrm{~h}$. The values of the parameter $f$ (fitting results) are given next to the corresponding curves.

result in a substantial reduction of the metabolic rate.

It has also been suggested that tumor growth and metastasis may be influenced by mechanical stress. In particular, Helmlinger et al. have investigated the volumetric growth of MTS cultured in agarose gels, showing that it is inhibited when the gel concentration is increased. ${ }^{13}$ This result can be explained with increasing local stress, which in turn leads to a higher cell (rather then mass) density $\rho$. Correspondingly, Freyer and Schor report that cells in the internal MTS layers experience a size reduction by up to a factor of $3{ }^{14}$ In our model we assume that MTS exhibit the ability to be compacted, if not compressed, under mechanical stress, so that $\rho$ varies (e.g., exponentially) from an initial value $\rho_{0}$ to an asymptotic value $\mathrm{R}$.

Defining the auxiliary variables: $s(t)=(v(t) / V)^{1-p}$ and $g(t)=(\rho(t) / R)^{1-p}$, their product " $s g$ " corresponds now to the "mass related" variable " $1-y$ " in Eq. (5). By time differentiation and making explicit use of Eq. (5) we obtain

$$
-\frac{d}{d t}(g s)=-g \frac{d s}{d t}-s \frac{d g}{d t}=\alpha f-\alpha(1-s g) \quad(5 b i s) .
$$

After rearranging the terms and inserting the variable $y$ one gets

$$
\frac{d y}{d t}=-\frac{d s}{d t}=\frac{(1-y)}{g} \frac{d g}{d t}+\alpha(1-y)+\frac{\alpha}{g(1-f)} \text { (5ter). }
$$

Finally, by noting that $1 / g d g / d t$ can be rewritten as $d(\ln g) / d t$, Eq. (5ter) becomes

$$
\frac{d y}{d t}+\left(\alpha+\frac{d \ln g}{d t}\right)(y-1)=-\frac{\alpha(1-f)}{g}\left(1-e^{-t / T}\right) .
$$

Here the parameter $f$ accounts for the effect of both the "missing energy input" (as before) and the mechanical stress.

Growth data for various gel concentrations from Ref. 13 are shown together with model fits in Fig. 2. Note the substantial increase in the accommodation time as the gel concentration (and thus the mechanical stress) is increased. These results suggest that it takes a relatively long time for 


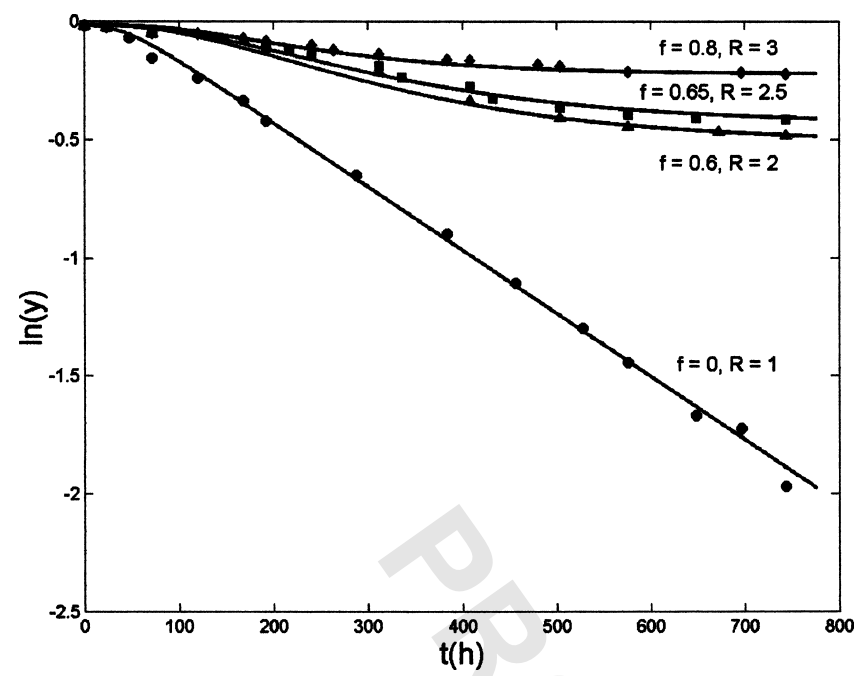

FIG. 2. Time variation of the spheroid volume growing under different mechanical stress conditions due to various gel concentrations [see Eqs. (6) and (7), data from Ref. 13]. Solid curves are model fits. The $y$ intercept corresponds to $v_{0}=4 \times 10^{-9} \mathrm{~cm}^{3}$. Final volumes and accommodation times $(V, T)$ are, starting from the lowest curve: $\left(6 \times 10^{-4} \mathrm{~cm}^{3}, 30 \mathrm{~h}\right),(3.8$ $\left.\times 10^{-5} \mathrm{~cm}^{3}, 100 \mathrm{~h}\right),\left(2.65 \times 10^{-5} \mathrm{~cm}^{3}, T=110 \mathrm{~h}\right),\left(4.88 \times 10^{-6} \mathrm{~cm}^{3}, 120 \mathrm{~h}\right)$. The values of $f$ and $R$ (fitting results) are given next to the corresponding curves.

the tumor cells to develop a suitable nutrient uptake within a stressed matrix. Finally, the results show that the effective undernourishment $f$ is increased with the gel concentration, while the terminal tumor volume is sharply reduced.

In here we suggest that multicellular tumor spheroids can be used as an experimental tool to test allometric growth laws under controlled environmental conditions of insufficient nutrient supply and rising mechanical stress. The exponent in Eq. (6) indicates that growth is less affected by malnourishment when $p$ is farther away from unity. Although we derived Eq. (5) for MTS, one could speculate that the final size of tumors that grow according to the 3/4 law will be more severely modified by nutrient scarcity than in those following the $2 / 3$ law. In two-dimensional (2D) in vitro diffusion-controlled growth, $p=2 / 3$ should be replaced by $p=\frac{1}{2}$ (if nutrient enters only through the rim), while for a linear cell array (i.e., growing in a tube and fed through its ends) we should assume $p=0$. This indicates that sensitivity to nutrient deprivation is likely to be decreased in lowerdimensionality tumor cell aggregates (e.g., for single inva- sive tumor cells forming chain-like patterns along preferred anatomically pathways in vivo). We note that our results do not apply to a 2D culture submerged in nutrients, for which Eq. (2) needs to be modified. Finally, our results for growth under stress indicate that normal nutrient uptake patterns are severely impaired by increasing mechanical stress. This should have implications for in vivo cancer growth in the neighborhood of robust tissues, such as bone.

We note that the current setup does not yet explicitly consider space nor does it account for necrosis and apoptosis, i.e., cell death in the tumor's center. In vivo, tumors often develop such a necrotic core once they reach a certain volume. We have not included necrosis at this stage because (i) it would make the data interpretation less transparent as new parameters must be added, and (ii) other than in vivo tumors, microscopic tumor spheroids often develop much smaller necrotic cores, if at all. However, introducing spatial heterogeneity may open new possibilities aiming at predictions of MTS' spatio-temporal evolution, both from macro- and mesoscopic reference scales.

Our conclusions should stimulate more such experimental studies in order to shed light not only on various aspects of the dynamics of cancer growth, but also on the current allometric growth law controversy.

Y.M. is the recipient of a NCI-Training Grant Fellowship from the National Institutes of Health (CA09502).

${ }^{1}$ C. Guiot, P. G. Degiorgis, P. P. Delsanto, P. Gabriele, and T. S. Deisboeck, J. Theor. Biol. 225,147 (2003).

${ }^{2}$ G. B. West, J. H. Brown, and B. J. Enquist, Nature (London) 413, 628 (2001).

${ }^{3}$ P. S. Dodds, D. H. Rothman, and J. S. Weitz, J. Theor. Biol. 209, 9 (2001). ${ }^{4}$ M. Martin, J. Natl. Cancer Inst. 95, 704 (2003).

${ }^{5}$ P. Cohen, New Sci. (2003).

${ }^{6}$ J. J. Blum, J. Theor. Biol. 64, 599 (1977).

${ }^{7}$ L. Demetrius, Physica A 322, 477 (2003).

${ }^{8}$ V. B. Kokshenev, Physica A 322, 491 (2003).

${ }^{9}$ O. Oudar, Crit. Rev. Oncol. Hematol. 36, 99 (2000).

${ }^{10}$ R. Chignola, A. Schenetti, E. Chiesa, R. Foroni, S. Sartoris, A. Brendolan, G. Tridente, G. Andrighetto, and D. Liberati, Cell Prolif 33, 219 (2000).

${ }^{11}$ M. Marusic, Z. Bajzer, S. Vuk-Pavlovic, and J. P. Freyer, Bull. Math. Biol. 56, 617 (1994); M. Marusic, Z. Bajzer, J. P. Freyer, and S. Vuc-Pavlovic, Cell Prolif 27, 73 (1994).

${ }^{12}$ J. P. Freyer, and R. M. Sutherland, Cancer Res. 46, 3504 (1986).

${ }^{13}$ G. Helmlinger, P. A. Netti, H. C. Lichtenbeld, R. J. Melder, and R. K. Jain, Nat. Biotechnol. 15, 778 (1997).

${ }^{14}$ J. P. Freyer and P. L. Schor, J. Cell Physiol. 138, 384 (1989). 